Research Article

\title{
Swelling Resistance and Water-Induced Shape Memory Performances of Sisal Cellulose Nanofibers/Polyethylene Glycol/Citric Acid Nanocellulose Papers
}

\author{
Zuocai Zhang $\mathbb{D}^{1},{ }^{1}$ Yuqi Li $\mathbb{D}^{1},{ }^{1}$ Laifu Song, ${ }^{1}$ Li Ren, ${ }^{1}$ Xu Xu, ${ }^{1,2}$ and Shaorong Lu $\mathbb{D}^{1}$ \\ ${ }^{1}$ Key Laboratory of New Processing Technology for Nonferrous Metals and Materials, Ministry of Education, School of Material \\ Science and Engineering, Guilin University of Technology, Guilin 541004, China \\ ${ }^{2}$ Department of Mechanical, Automotive \& Materials Engineering, University of Windsor, 401 Sunset Ave, Windsor, ON, Canada \\ N9B $3 P 4$
}

Correspondence should be addressed to Yuqi Li; liyuqi@glut.edu.cn and Shaorong Lu; lushaor@163.com

Received 4 March 2019; Revised 4 May 2019; Accepted 23 May 2019; Published 22 July 2019

Guest Editor: Biaolin Peng

Copyright (C) 2019 Zuocai Zhang et al. This is an open access article distributed under the Creative Commons Attribution License, which permits unrestricted use, distribution, and reproduction in any medium, provided the original work is properly cited.

In this work, a kind of nanocomposite paper was obtained by evaporation-induced self-assembly of a mixture of sisal cellulose nanofibers (CNF) and polyethylene glycol (PEG) as the matrix and citric acid (CA) as a cross-linking agent. The CNF/PEG/CA paper exhibited good water swelling resistance which could be controlled by changing the concentration of CA. In addition, this nanocomposite paper exhibited good mechanical properties and water-induced shape memory performance. In particular, when the dosage of CA was $30 \mathrm{wt} . \%$, the tensile strength and the tensile modulus of the CNF/PEG/CA paper after swelling were 25.2 $\mathrm{MPa}$ and $813.0 \mathrm{MPa}$, respectively. Further, this nanocomposite showed great potential for water-induced shape memory materials with fast response speed. The shape recovery rate ( $\mathrm{Rr}$ ) of the CNF/PEG/CA paper reached $90.2 \%$ with 30 wt.\% CA after being immersed in water for $11 \mathrm{~s}$. It is anticipated that our current work can be used to exploit more efficient methods to overcome the poor water swelling resistance of the cellulose-based shape memory materials.

\section{Introduction}

Shape memory polymer is a new type of intelligent material, as well as the most important part of intelligent materials. Shape memory polymer can reflect the shape memory performance mainly due to its internal molecular structure. Its molecular structure is a kind of network usually composed of reversible phase and stationary phase. The stationary phase can be a cross-linked node by chemical reaction or physical reaction, and it plays an important role in building a cross-linking network $[1,2]$. The reversible phase is usually glassy-amorphous transformation, crystallization-melting transformation, etc., and the phase transition is also varied. Shape memory polymer composites are widely used in many fields, such as drug release material, smart textiles, structural materials, sensors, and fiber optic solar cells [3-5]. With the rapid development of science and technology, most of the shape memory polymers are thermotropic memory mate- rials, such as polycaprolactone (PCL), polyurethane (PU), polylactic acid (PLA), and epoxy resin (EP) [6-10]. Usually, these polymers are network structure formed by covalent cross-linking, and their shape recovery temperature is higher when compared with other common polymers, which leads to limitations in biomedicine or some cryogenic applications [11]. In medicine fields, a key challenge is that an excess of heat usually causes damage to biological tissue. Nevertheless, water-induced shape memory polymers can effectively compensate for this deficiency [12]. Based on the reported waterinduced shape memory polymer, most of them are made from petroleum chemical raw materials, which are not renewable, biodegradable, and biocompatible $[13,14]$.

Cellulose is a kind of natural polymer with green environmental protection, abundant source, and unique layered structure [15]. In recent years, the extraction of nanofiber from biomass raw materials is used as a research hotspot for the enhancement of nanocomposite [16-18]. Due to the 
nanometer size effect and high specific surface area of nanocellulose, the mechanical properties of nanocomposite are much higher than those of conventional composites [19, 20]. In addition, the nanocellulose with high mechanical strength, high length-diameter ratio, and low price and degradable is environmentally friendly, which is harmless to humans and animals [21]. Nanocellulose contains a lot of hydroxyl. Using the function of hydrogen bonds, it can be used to synthesize the shape memory polymer with water response effect $[18,22]$, biodegradability $[23,24]$, and biocompatibility [25]. However, the nanocomposite material simply combined by the Van der Waals force has a larger volume of swelling and a higher rate of water absorption after soaking in water [16]. Nevertheless, in the wet state, its mechanical properties greatly reduce, limiting its application in certain areas.

Polyethylene glycol (PEG) is a kind of thermoplastic polymer material with high crystallinity and good water solubility, is nontoxic, and is nonirritating, and it has great toughness and good biocompatibility $[12,26]$, making it a good choice to be used as another kind of flexible chain segment of the interpenetrating network structure [27]. Therefore, based on the advantage of the cellulose nanofibers (CNF) with PEG, the CNF and PEG were cross-linked to form a new network shape memory nanocomposite (CNF/PEG/CA) paper, through citric acid (CA) as a crosslinking agent [28].

The CNF/PEG/CA paper was prepared by the method of evaporation-induced self-assembly (EISA) [29]. The preparation is easy to operate, fast, green environmental, and has high extraction rate. The formation mechanism of the $\mathrm{CNF} / \mathrm{PEG} / \mathrm{CA}$ interpenetrating network structure shape memory nanocomposite paper is shown in Figure 1. The mechanical strength is poor, and the swelling resistance is low when the nanocellulose materials simply are combined by the Van der Waals force under the wetting state. CA can combine cellulose molecules and PEG molecules through covalent bond connection to form an interpenetrating network structure, which improves the performance of the shape memory nanocomposite paper $[30,31]$. The flow chart shows the process to prepare the CNF/PEG/CA interpenetrating network shape memory nanocomposite paper by EISA. In addition, the shape and the size of the $\mathrm{CNF} / \mathrm{PEG} / \mathrm{CA}$ paper can be adjusted by the forming mold. And the CNF/PEG/CA paper has good flexibility; as a result, it can be folded into various shapes. The CNF/PEG/CA paper has good hydrophilicity, biocompatibility, and biodegradability and keeps good mechanical properties after swelling by absorbing water, which leads to the CNF/PEG/CA paper having wide application prospection in the biomedical field.

\section{Experimental}

Sisal fibers were bought from Guangxi Sisal Company, China. $\mathrm{NaOH}, \mathrm{CH}_{3} \mathrm{COOH}$, citric acid (CA), and $\mathrm{NaClO}_{2}$ were purchased from Aladdin Chemistry Co. Ltd. Polyethylene glycol 1000 (PEG) and $\mathrm{Na}_{2} \mathrm{SO}_{4} \cdot 10 \mathrm{H}_{2} \mathrm{O}$ were supplied by Sinopharm Chemical Reagent. The other chemicals used were all analytical pure reagents without further purification.
2.1. Preparation of Microcrystalline Sisal Fibers (MSF). Firstly, sisal fibers were cut into an approximate length of $5 \mathrm{~cm}$ and the lignin and the pectin particles on the surface of sisal fibers were washed away by deionized water. Then, the sisal fibers were dried in an oven at $60^{\circ} \mathrm{C}$ for later use. The $50 \mathrm{~g}$ dry fibers were boiled in aqueous solution of $14.0 \mathrm{~g} \mathrm{NaOH}, 14.0 \mathrm{~g} \mathrm{Na}_{2} \mathrm{SO}_{4} \cdot 10 \mathrm{H}_{2} \mathrm{O}$, and $400 \mathrm{ml}$ deionized water in $500 \mathrm{ml}$ autoclaves at $170^{\circ} \mathrm{C}$ for $2 \mathrm{~h}$. After the reaction, the sisal fibers were filtered and washed several times with deionized water until the solution remains the same color, then dried in an oven at $60^{\circ} \mathrm{C}$. Later, the $20 \mathrm{~g}$ dry fibers were transferred to a $1000 \mathrm{ml}$ three-necked flask, adding $6.7 \mathrm{~g} \mathrm{NaClO}_{2}, 5 \mathrm{ml} \mathrm{CH}_{3} \mathrm{COOH}$, and $650 \mathrm{ml}$ deionized water, and the mixture kept reacting at $75-80^{\circ} \mathrm{C}$ for $2-3 \mathrm{~h}$ to bleach. The product was filtered and washed with water after the reaction. Finally, the MSF were dried in an oven at $60^{\circ} \mathrm{C}$ until constant weight [16].

2.2. Preparation of Sisal Cellulose Nanofibers (CNF). Firstly, the $12.0 \mathrm{~g}$ dried MSF were soaked wet in $40 \mathrm{ml}$ preconfigured aqueous solution of $\mathrm{NaOH}(10 \mathrm{wt} . \%)$ and transferred to the $500 \mathrm{ml}$ three-necked round bottomed flask. Then, $350 \mathrm{ml}$ absolute ethyl alcohol was added into the flask under mechanical agitation for $30 \mathrm{~min}$ to make it evenly dispersed and alkalified. After that, $6 \mathrm{~g}$ chloroacetic acid $\left(\mathrm{CH}_{2} \mathrm{ClCOOH}\right)$ was added to the mixture, and the reaction was at the temperature of $70^{\circ} \mathrm{C}$ for $3 \mathrm{~h}$ under mechanical stirring. At the end of the reaction, the crude product was collected after filtering the mixture, repeated centrifugal washing with distilled water to remove unreacted chloroacetic acid until the $\mathrm{pH}$ value was neutral, and drying. The crude product was mixed with a certain amount of deionized water to make its solid content at about $0.5 \mathrm{wt} . \%$, then the mixture was sheared by a high-shear dispersion homogenizer at $28000 \mathrm{r} / \mathrm{min}$ high speed for $30 \mathrm{~min}$ to get the transparent jelly product. After using a 400-mesh filter cloth to extrude the product to preliminary filtrate the unsegregated crude fiber, adjusting proportional to about $0.1 \mathrm{wt} . \%$ with deionized water and centrifuging for $10 \mathrm{~min}$ at $12000 \mathrm{r} / \mathrm{min}$, the CNF with pale blue were obtained from the centrifugal barrel upper $80 \%$ solution, at last rotating enrichment and measuring its solid content to set aside.

2.3. Preparation of Shape Memory Nanocomposite (CNF/PEG/CA) Paper. The detailed fabrication procedure of the CNF/PEG/CA paper was as follows. The CNF were configured to $5 \mathrm{mg} / \mathrm{ml}$ aqueous dispersion with deionized water, and a certain amount of PEG was weighed to dissolve in $5 \mathrm{ml}$ deionized water at the same time. Then, the abovementioned two kinds of solutions were mixed with continuous stirring and ultrasonic dispersion for $30 \mathrm{~min}$ to make it even. After adding a certain amount of CA as a crosslinking agent, stirring for $10 \mathrm{~min}$ to mix evenly, the mixture was poured into the tetrafluoroethylene mold and transferred to the drum wind drying oven at $60^{\circ} \mathrm{C}$ for $24 \mathrm{~h}$. By evaporating the moisture slowly, CNF and PEG molecules were crosslinked by CA molecules to be assembled into a CNF/PEG/CA paper with interpenetrating network structures. The thickness and size of the CNF/PEG/CA paper interpenetrating 


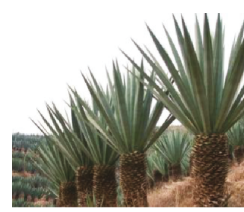

Sisal fiber

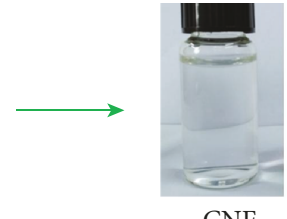

$\mathrm{CNF}$
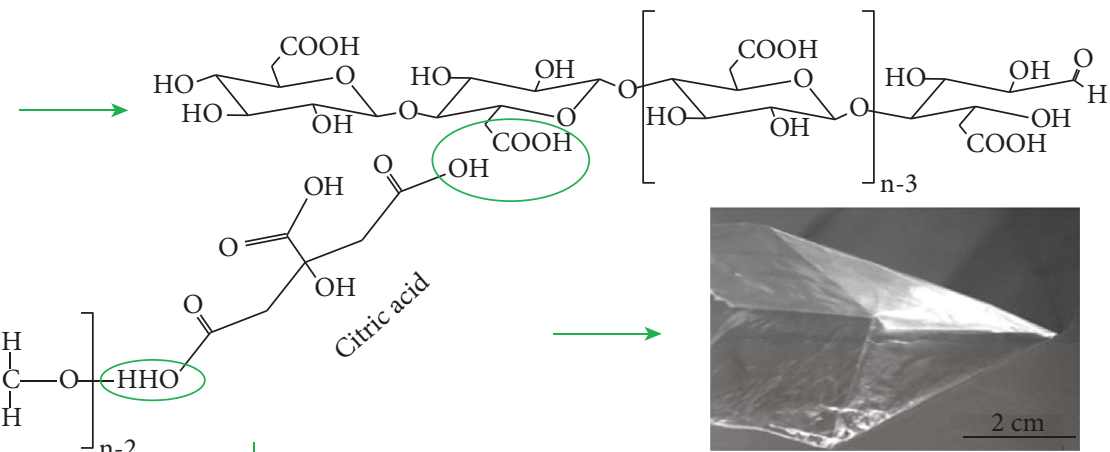

CNF/PEG/CA paper

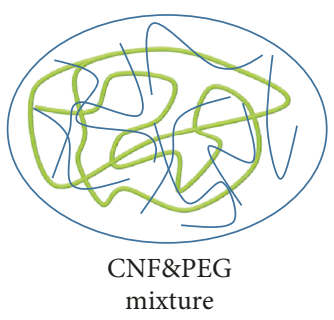

CA
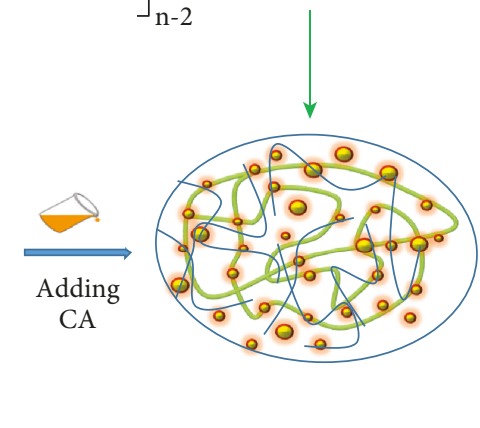<smiles>C=CC(C)(O)CC(C)C</smiles><smiles>C1CC2C[C+]1C2</smiles><smiles>C1CC2CCOC2C1</smiles><smiles>OC1CCCC1</smiles>
Polyethylene glycol $\mathrm{n}-2$
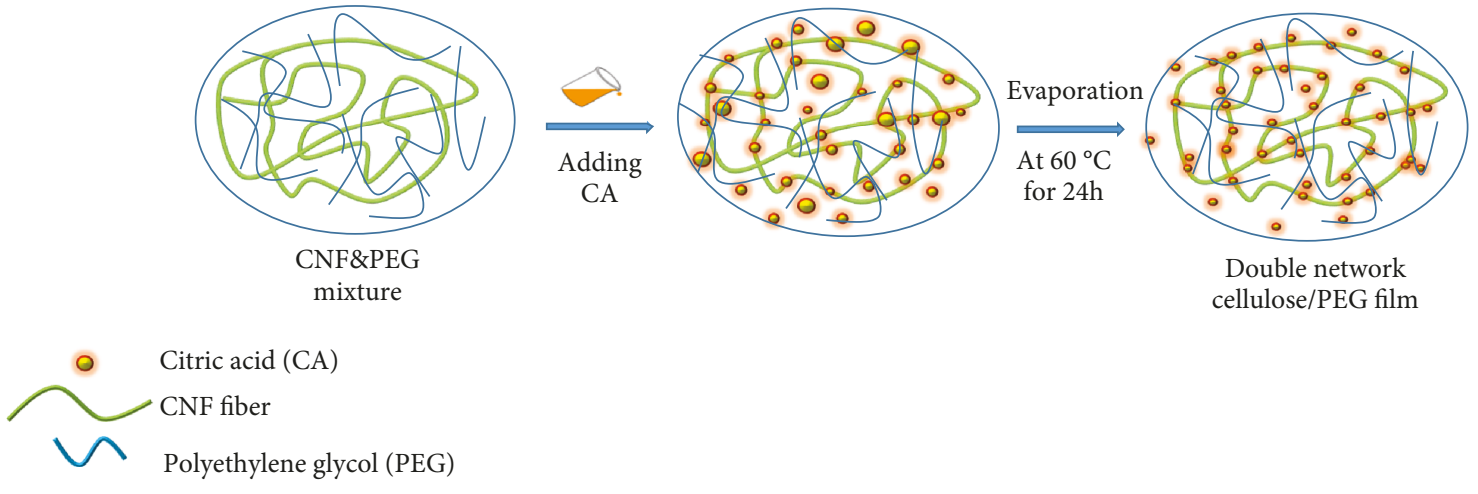

FIgURe 1: Preparation process of the CNF/PEG/CA paper.

network composite structure can be controlled by the amount of mixed solution and the size of the mold. To study the influence of different contents of the CA cross-linking agent on the interpenetrating network CNF/PEG/CA paper, we prepared different cross-linking degrees of interpenetrating network CNF/PEG/CA paper according to the formula design of Table 1.

2.4. Characterization. The infrared spectrum of the sample prepared by $\mathrm{KBr}$ tableting was analyzed by the American Nicolet Nexus 470 type Fourier transformation infrared spectrometer (spectra range: $4000 \sim 500 \mathrm{~cm}^{-1}$ ). The water swelling resistance of the shape memory nanocomposite paper was characterized by the change of the volume and the weight before and after immersion in deionized water at $25^{\circ} \mathrm{C}$ for $5 \mathrm{~min}$. According to the water absorption $(\%)=($ $\left.M_{1}-M_{0}\right) / M_{0} \times 100, \quad$ swelling rate $=\left(V_{1}-V_{0}\right) / V_{0} \times 100 \%$, where $M_{0}$ and $V_{0}$ are the quantity and the volume of the sample, respectively, before absorbing water, and $M_{1}$ and $V_{1}$ are the quantity and the volume of the sample, respectively, after absorbing water. All of the samples were cut into the size of $30 \mathrm{~mm} \times 3 \mathrm{~mm} \times 0.05 \mathrm{~mm}$, and the text results were averaged after each group of samples was measured three times. The tensile strength was examined on a Q800 dynamic mechanical analyzer (TA Instruments, USA) by stretching control at the temperature of $25^{\circ} \mathrm{C}$ and a rate of $3 \mathrm{~N} \mathrm{~min}^{-1}$. The sample size was $30 \mathrm{~mm} \times 3 \mathrm{~mm} \times 0.05 \mathrm{~mm}$, and the arithmetic average of the tensile strength test of 5
TABLE 1: The blend design of compounding materials for respective composites.

\begin{tabular}{lccc}
\hline Nanocomposite & CNF (wt.\%) & PEG (wt.\%) & CA (wt.\%) \\
\hline CNF/PEG/CA & 70 & 30 & 0 \\
CNF/PEG/CA-1 & 70 & 30 & 10 \\
CNF/PEG/CA-2 & 70 & 30 & 20 \\
CNF/PEG/CA-3 & 70 & 30 & 30 \\
CNF/PEG/CA-4 & 70 & 30 & 40 \\
\hline
\end{tabular}

samples was recorded for the tensile properties of the sample. The sample cross section was sputter-coated with gold in case of electronic accumulation and then tested by scanning electron microscopy (JSM-6480LV, Japan JEOL) at an accelerating voltage of $3.0 \mathrm{kV}$. The gel content of the sample $(G)$ was measured by the mass (miso) of the sample before extraction in methylene chloride as extracting agent for $24 \mathrm{~h}$ of Soxhlet extraction and the mass of the dried sample $\left(m_{\mathrm{d}}\right)$. According to the following formula, $G=m_{\mathrm{d}} / \mathrm{miso}$ to calculate the gel content of the material. The water-induced shape memory effect was investigated as follows. The specimen was folded to a temporary shape at $60^{\circ} \mathrm{C}$ and cooled down to $0^{\circ} \mathrm{C}$ with the external force to fix the temporary shape. The specimen was immersed in water at $25^{\circ} \mathrm{C}$ to determine the recovery of shape, and the above two processes were recorded by a video camera. The recovery ratio was defined as $\operatorname{Rr}=\left[\left(\theta_{\mathrm{f}}-\theta_{0}\right) /\left(180-\theta_{0}\right)\right] \times 100 \%$, where $\mathrm{Rr}$ represents 
the response rate, $\theta_{\mathrm{f}}$ represents the recovery angle of sample at a different time, and $\theta_{0}$ represents the angle of the sample before recovering. All the presented results were an average of three specimens with the size of $30 \mathrm{~mm} \times 3 \mathrm{~mm} \times 0.05$ $\mathrm{mm}$ (length $\times$ width $\times$ thickness) [16]. The light transmittance of shape memory nanocomposite paper mainly adopted the double beam spectrophotometer (UV-3600NIR, Shimadzu) with wavelength range $200-800 \mathrm{~nm}$, scan rate $300 \mathrm{~nm} / \mathrm{min}$, sampling interval $1 \mathrm{~nm}$, test temperature $25^{\circ} \mathrm{C}$, and $100 \%$ sensitivity. Each sample was tested three times to ensure the accuracy of the test data.

\section{Results and Discussion}

\subsection{FT-IR Spectra of CNF, PEG, CA, and CNF/PEG/CA} Paper. As shown in Figure 2(a), there are two vibration absorption peaks at $3413 \mathrm{~cm}^{-1}$ and $2919 \mathrm{~cm}^{-1}$, respectively, belonging to the stretching vibration of $-\mathrm{OH}$ and $-\mathrm{CH}-$ on the CNF molecular chains. Additionally, the two vibration absorption peaks at $1599 \mathrm{~cm}^{-1}$ and $1382 \mathrm{~cm}^{-1}$ can be found, which refer to the symmetric stretching vibration and the antisymmetric stretching vibration of carboxylic acid sodium salt groups on cellulose, respectively [16]. It illustrates that the CNF are mainly in the form of carboxylic acid sodium after being treated with chloroacetic acid. In the FT-IR curve of PEG, the absorption peaks at $3430 \mathrm{~cm}^{-1}, 2872 \mathrm{~cm}^{-1}$, and $1105 \mathrm{~cm}^{-1}$ refer to the stretching vibration of $-\mathrm{OH}$ and - $\mathrm{CH}$ - and $\mathrm{C}-\mathrm{O}-\mathrm{C}$, respectively. From the infrared spectrum of CA [30], it can be seen that there are two strong absorption peaks at $1688 \mathrm{~cm}^{-1}$ and $1757 \mathrm{~cm}^{-1}$, which belong to the $\mathrm{C}=\mathrm{O}$ stretching vibration on CA. From Figure 2(b), the -OH vibration absorption of the CNF/PEG/CA paper at $3410 \mathrm{~cm}^{-1}$ decreases obviously with the increase of CA adding amount. According to the changes in the FT-IR spectra, it can be found that the PEG and CNF can be effectively cross-linked together by CA.

3.2. Water Resistance of the CNF/PEG and CNF/PEG/CA Papers. To study the water swelling resistance of the $\mathrm{CNF} / \mathrm{PEG}$ and CNF/PEG/CA paper, the water absorption and swelling rate change with the adding of CA was investigated. As shown in Figure 3, the water absorption and swelling rate of CNF/PEG paper are quite high. The water absorption is up to $1038 \%$ and the swelling rate is up to $2100 \%$. This is mainly due to the fact that the surface of $\mathrm{CNF}$ contains a lot of $-\mathrm{OH}$ and $-\mathrm{COOH}$ - hydrophilic groups, leading to high hydrophilicity. By contrast, the water absorption and the volume swelling rate of the CNF/PEG/CA paper are decreased greatly with the increase of CA adding amount. At CA 30 wt.\%, the water absorption and swelling rate of the $\mathrm{CNF} / \mathrm{PEG} / \mathrm{CA}$ paper are decreased 46 times and 15 times, respectively, compared to those of the CNF/PEG paper. The reason for this is that the CNF and PEG are crosslinked by CA, leading to the increase of hard segment area in the CNF/PEG/CA paper. In addition, hydrophilic groups such as hydroxyl on the CNF and PEG can react with CA. Therefore, the water absorption and the swelling rate of $\mathrm{CNF} / \mathrm{PEG} / \mathrm{CA}$ paper reduced.
Gel content $(G)$ is used to study the cross-linking degree of the CNF/PEG/CA paper. From Figure 4, the $G$ value of the $\mathrm{CNF} / \mathrm{PEG}$ paper without CA cross-linking is lower, which is about $74 \%$. When adding $C A$, the $G$ value of the $\mathrm{CNF} / \mathrm{PEG} / \mathrm{CA}$ paper gradually increases with the addition of CA. When adding $30 \mathrm{wt} \% \mathrm{CA}$, the $G$ value of the $\mathrm{CNF} / \mathrm{PEG} / \mathrm{CA}$ paper tends to be stable, reaching $90 \%$, and the $G$ value of the CNF/PEG paper increases by $21.6 \%$ compared with the CNF/PEG paper without CA cross-linking. It indicates that the addition of CA is effective to cross-link PEG and CNF, to form a stable network structure.

3.3. Mechanical Properties of the CNF/PEG and CNF/PEG/CA Papers. Water-induced shape memory materials are mainly limited by the decrease in mechanical properties after swelling. Figure 5 shows the mechanical properties of the CNF/PEG/CA paper with different CA contents before being soaked in water. As shown in Figure 5(a), the elongation at break of the CNF/PEG/CA paper decreases with the content of CA increasing, because of the increasing of the cross-linking degree. As shown in Figure 5(b), with the increase of CA contents, the tensile strength of the CNF/PEG/CA paper shows a downward trend, while the tensile modulus increases. When adding $30 \mathrm{wt} . \% \mathrm{CA}$, the tensile modulus of the CNF/PEG/CA paper increases from $853 \mathrm{MPa}$ to $1401 \mathrm{MPa}$. It can be attributed to the formation of strong covalent bonds, formed by the esterification reaction of carboxyl on CA and hydroxyl groups on PEG and CNF cellulose, which improves the stability of the molecular chain. In addition, the hydroxyl groups on CNF and PEG decrease, so the effect of hydrogen bonding between molecules greatly reduces, which makes it difficult for the movement of chain segments. Therefore, reducing the CNF/PEG/CA paper plastic deformation, the tensile strength and the elongation at break all decrease, and the tensile modulus greatly improves.

Figure 6(a) shows the mechanical properties of the CNF/PEG/CA paper with different CA contents after being soaked in water. It can be seen that the CNF/PEG paper without CA cross-linking exhibits quite poor mechanical properties after being soaked in water. It can be attributed to the combination of the internal molecules of the CNF/PEG paper, which is mainly formed by hydrogen bonding and the molecular chain entanglement between molecular chains without CA cross-linking. When water immerse into the interior of the material, the hydrogen bonding force between the CNF and PEG molecules is damaged, which decreases the mechanical properties. In contrast, as shown in Figure 6(b), the tensile strength and the tensile modulus of the wet $\mathrm{CNF} / \mathrm{PEG} / \mathrm{CA}$ paper increase greatly with the increasing of CA contents. When the addition amount of CA is $30 \mathrm{wt} . \%$, the tensile strength and tensile modulus of the CNF/PEG/CA paper after swelling are $25.2 \mathrm{MPa}$ and $813.0 \mathrm{MPa}$, respectively. It is the result that demonstrates that the chemical cross-linking network structure among PEG, CNF, and CA leads to good mechanical properties of the CNF/PEG/CA paper after soaking. Therefore, the CNF/PEG/CA paper is more suitable for underwater applications than other shape memory polymers. 


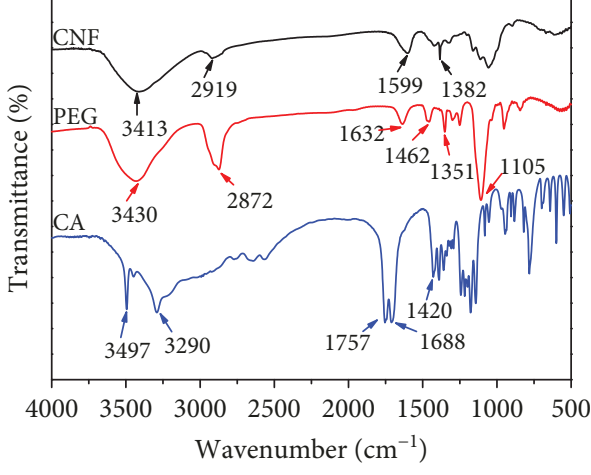

(a)

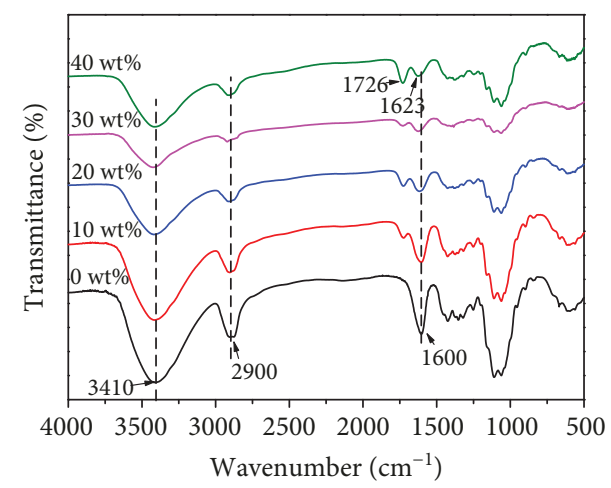

(b)

Figure 2: (a) The FT-IR spectra of CNF, PEG, CA, and (b) CNF/PEG/CA paper with different contents of CA.

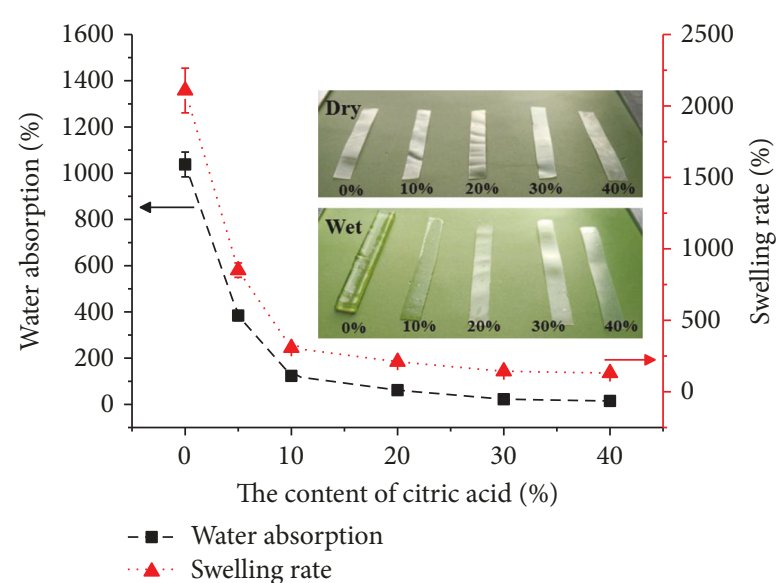

FIgURE 3: Water absorption and swelling rate of the CNF/PEG/CA paper; inset: the morphology of the CNF/PEG/CA paper before and after soaking.

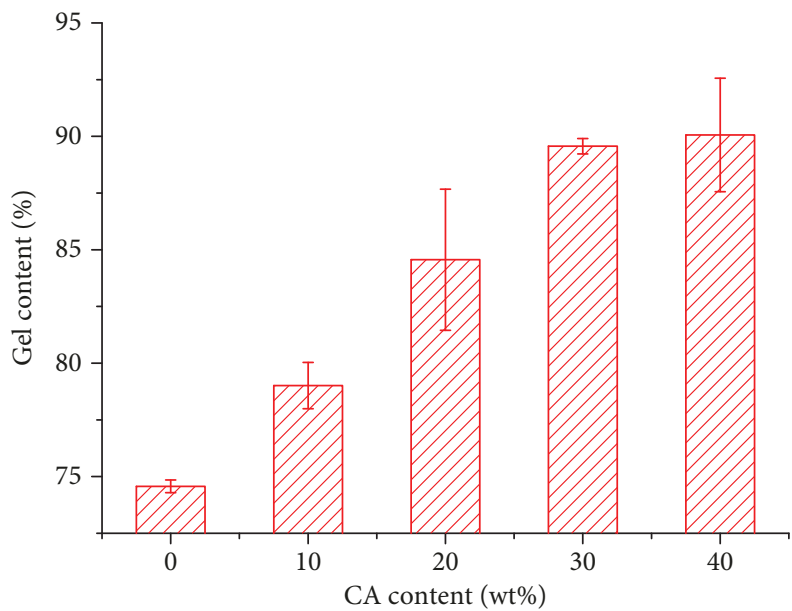

Figure 4: The gel content of the CNF/PEG and CNF/PEG/CA papers.
To microscopically analyze the mechanical performance, the scanning electron microscope (SEM) images of the fracture surfaces of the CNF/PEG/CA paper are used to investigate the interaction between $\mathrm{CA}$ and the $\mathrm{CNF} / \mathrm{PEG}$ paper. As shown in Figure 7(a), it can be seen that the section of the CNF/PEG paper without CA cross-linking is level and close and exhibits orientation arrangement in the vertical direction without obvious holes. It is attributed to the good compatibility between nanocellulose and PEG, due to the hydrogen bonding and mutual physical tangles. Therefore, the direction of fracture is consistent with the direction of force. As shown in Figures 7(b) and 7(c), the sections of the $\mathrm{CNF} / \mathrm{PEG} / \mathrm{CA}$ paper become rough after adding the crosslinking agent CA. In addition, there are many fold structures and interpenetrating holes, which are mainly due to the cross-linking between the carboxyl on CA and the hydroxyl on CNF cellulose and PEG that forms the permanent area of hard segments.

3.4. Water-Induced Shape Memory of the CNF/PEG and CNF/PEG/CA Papers. Figure 8 shows the shape recovery processes of the CNF/PEG/CA paper with different CA amounts. It can be seen that the water response of the $\mathrm{CNF} / \mathrm{PEG}$ paper without $\mathrm{CA}$ in $25^{\circ} \mathrm{C}$ water is fast, and the response rate is high. It illustrates that the CNF/PEG/CA paper has good water response performance. As shown in Figure 9, the water-induced shape performance of the CNF/PEG/CA paper with CA exhibits good water-induced shape memory performance as well. When the CA addition amount is up to $20 \mathrm{wt} . \%$, the shape recovery rate $(\mathrm{Rr})$ immersed in water for $10 \mathrm{~s}$ can reach over $99 \%$. At CA $30 \mathrm{wt} . \%, \mathrm{Rr}$ of the CNF/PEG/CA paper is about $90.2 \%$. The reason why the response rate reduces mainly is that the $\mathrm{CNF} / \mathrm{PEG} / \mathrm{CA}$ paper is based on hydrogen bonding water response to form memory switch. Additionally, the CNF and PEG molecular chain is fixed by CA, which may form the cross-linked structure. And with the increase of CA amount, the cross-linking degree increases. Thus, the amount of the hydrogen bonds between the molecules greatly reduces, greatly reducing the intermolecular hydrogen bond binding force, and the memory effect performance decreases. The main reason for this phenomenon is that the response 


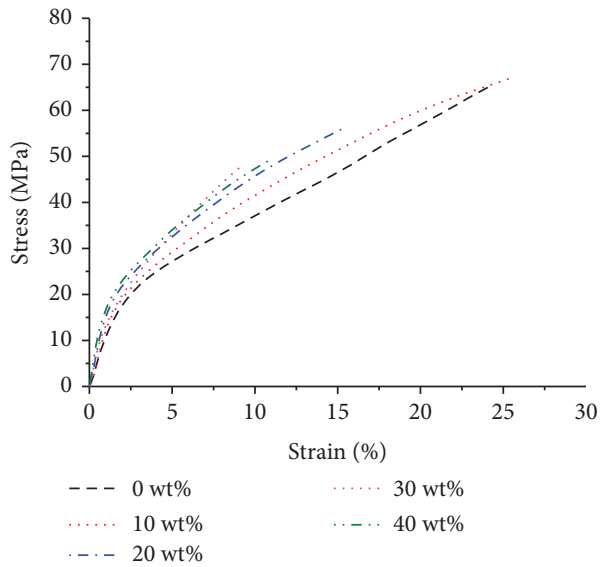

(a)

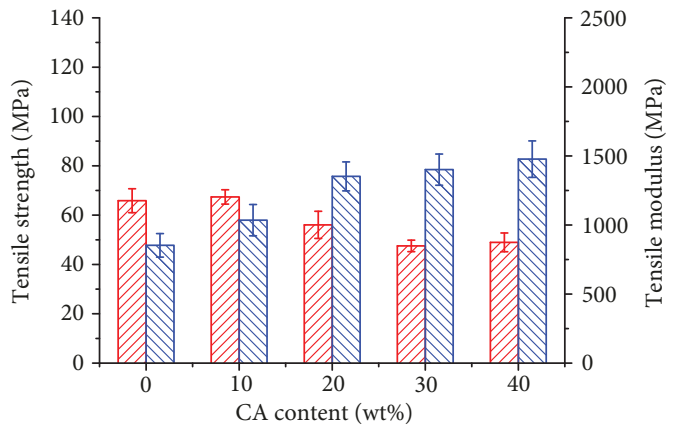

VIIA Tensile strength

MV Tensile modulus

(b)

FIGURE 5: (a) Typical stress-strain curves of the CNF/PEG/CA paper. (b) Tensile strength and modulus of the CNF/PEG/CA paper (before soaking).

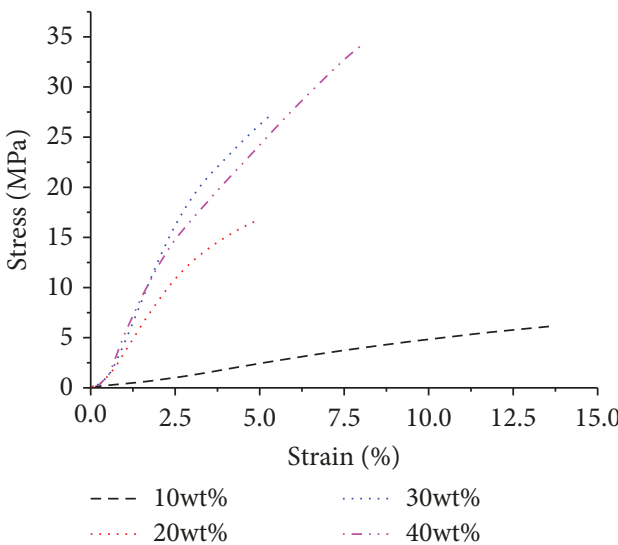

(a)

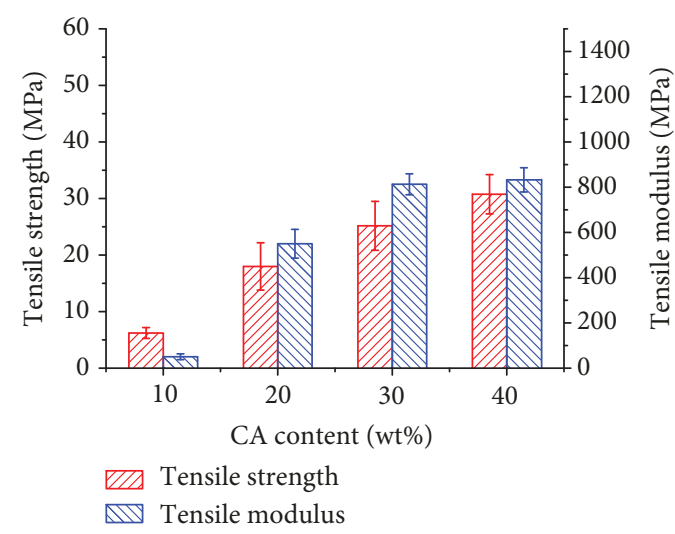

(b)

Figure 6: (a) Typical stress-strain curves of the CNF/PEG/CA paper. (b) Tensile strength and modulus of the CNF/PEG/CA paper (after soaking).

mechanism of soluble-induced shape memory materials is different from the thermal-induced shape memory materials [32-34]. The driving force of soluble-induced recovery is the plasticization of solvent molecule. As the solvent molecule enters the polymer network gradually, the transition temperature of the reversible phase gradually decreases, resulting in the phase transition at room temperature and driving the shape recovery of the material. Therefore, the solubleinduced recovery rate depends on the diffusion rate of the solvent molecule into the polymer network, and the diffusion rate of solvent molecules is related to the free volume of the polymer. For the CNF/PEG/CA interpenetrating network polymer, the larger the cross-linking degree is, the smaller the free volume will be, resulting in a lower diffusion rate of the solvent molecule and a slower recovery rate.

A water response mechanism of the CNF/PEG/CA paper was proposed in Figure 10. It can be seen that the CNF/PEG paper is formed by the intermolecular hydrogen bond selfassembly and has a certain interpenetrating network struc- ture. After adding CA, it embeds in the CNF/PEG paper substrate, and the carboxyl on CA and the hydroxyl on the CNF/PEG paper can be combined by esterification, which can form the interpenetrating network structure with a certain of rigidity. The deformation of the CNF/PEG/CA paper is temporary fixed by a strong hydrogen bond force between polymer molecules. When the CNF/PEG/CA paper is in water environment, water molecules enter in the interior of the $\mathrm{CNF} / \mathrm{PEG} / \mathrm{CA}$ interpenetrating network structure. The strong hydrogen bonds between molecules are broken, replaced by the weaker hydrogen bonds formed by water and polymer molecules. Thus, a force is released which fixes the temporary deformation, and the CNF/PEG/CA paper begins to recover from the temporary shape to the initial shape.

\section{Conclusions}

Shape memory nanocomposite (CNF/PEG/CA) paper was successfully prepared by the evaporation-induced self- 


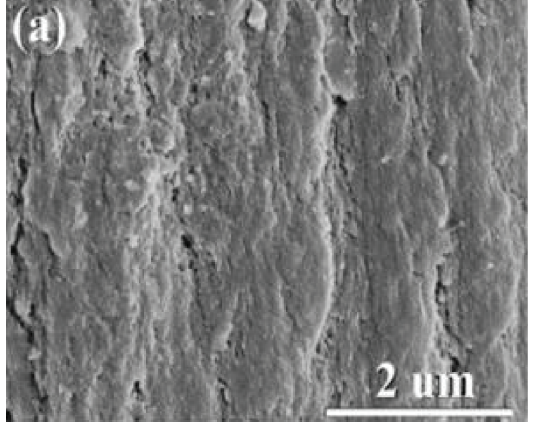

(a)

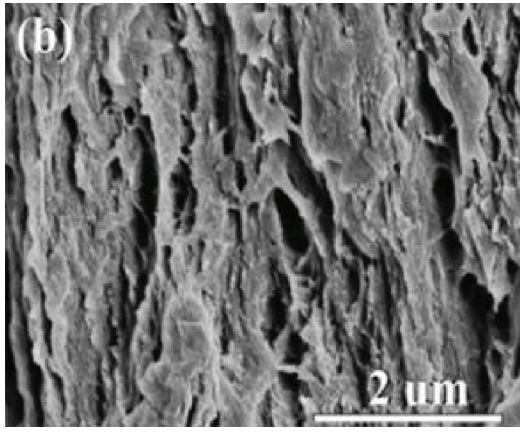

(b)

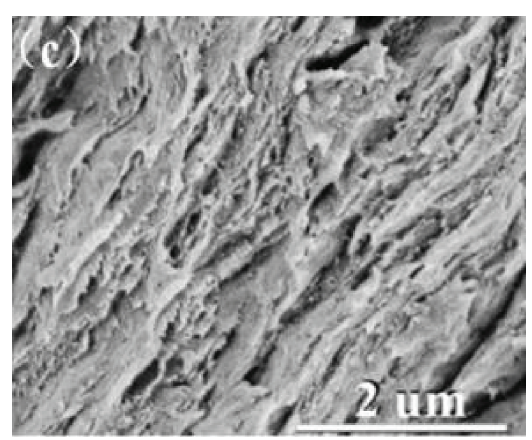

(c)

FIGURE 7: SEM images of the fractured surface of the neat CNF/PEG paper (a). CNF/PEG/CA paper with the CA content of 20 wt.\% (b) and $30 \mathrm{wt} . \%$ (c).
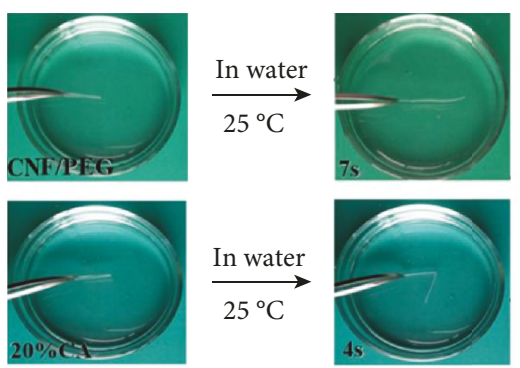

$$
\underset{25{ }^{\circ} \mathrm{C}}{\stackrel{\text { In water }}{\longrightarrow}}
$$

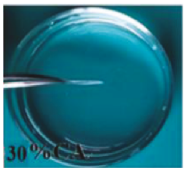

$\underset{25{ }^{\circ} \mathrm{C}}{\stackrel{\text { In water }}{\longrightarrow}}$
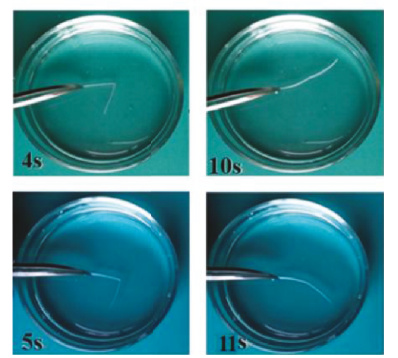

Figure 8: The shape recovery process of the CNF/PEG/CA paper.

assembly method, using sisal cellulose nanofibers (CNF) and polyethylene glycol (PEG) as the matrix and citric acid (CA) as a cross-linking agent. The results show that the CNF/PEG/CA paper exhibits good mechanical properties and water-induced shape memory performance. In particular, at CA 30 wt.\%, the tensile strength and the tensile modulus of the CNF/PEG/CA paper after swelling are 25.2 MPa and $813.0 \mathrm{MPa}$, respectively. Further, this nanocomposite has great potential for water-induced shape memory materials with fast response speed. Only for $11 \mathrm{~s}$, the shape recovery rate (Rr) of the CNF/PEG/CA paper with $30 \mathrm{wt} . \%$ of CA is $90.2 \%$ after being immersed in water. In addition, the

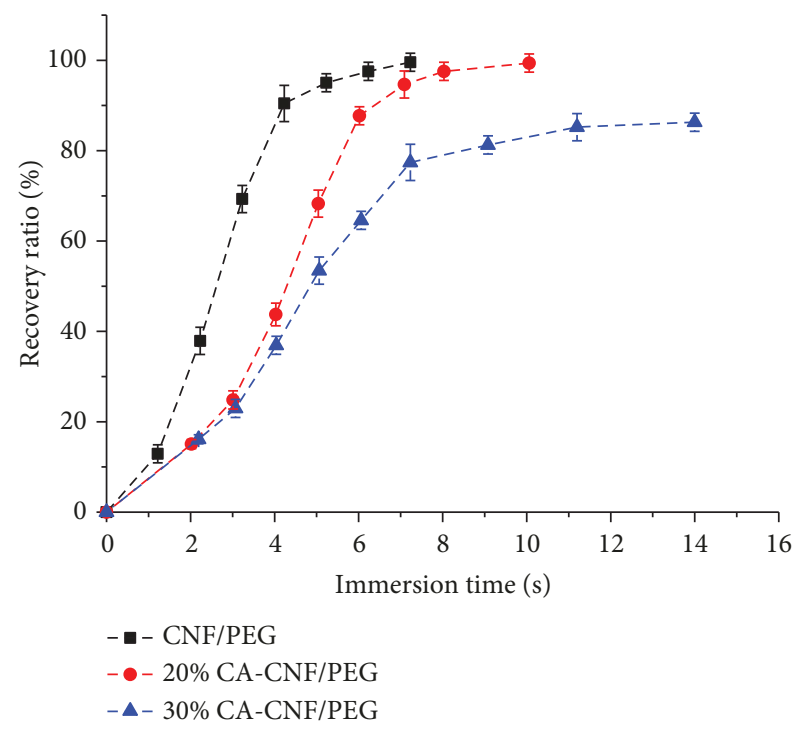

Figure 9: The shape memory behavior of the CNF/PEG/CA paper immersed in water.

CNF/PEG/CA paper has excellent water swelling resistance which could be controlled by changing the concentration of CA. After wet swelling, the highest tensile strength and tensile modulus are up to $30 \mathrm{MPa}$ and $832 \mathrm{MPa}$, respectively. It is hoped that this work can be used to exploit more efficient methods to overcome the poor water swelling resistance of the cellulose-based shape memory materials. 

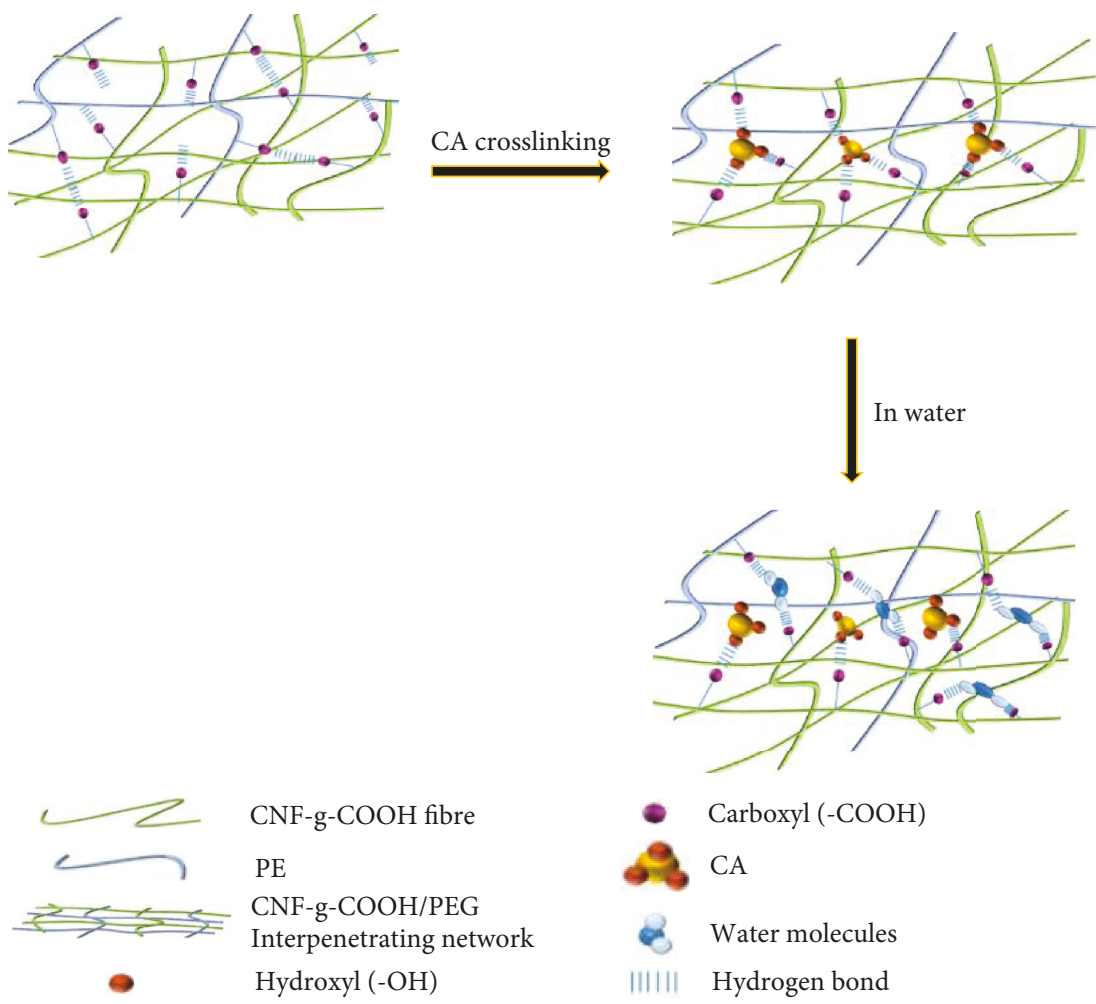

Figure 10: Schematic of the water-induced shape memory effect mechanism of the CNF/PEG/CA paper.

\section{Data Availability}

The data used to support the findings of this study are available from the corresponding authors upon request.

\section{Conflicts of Interest}

The authors declare no conflict of interest.

\section{Authors' Contributions}

Zuocai Zhang, Yuqi Li, and Shaorong Lu contributed equally to this work.

\section{Acknowledgments}

The authors gratefully acknowledge the financial support of the National Natural Science Foundation of China (51463007, 51605109, and 51763009), the Natural Science Foundation of Guangxi Province, China (2014GXNSFDA 118006 and 2016GXNSFBA380004), the Project of the Department of Science and Technology of Guilin (No. 2016012005), and the Young Teachers-Based Capacity Building Project of Guangxi (2018KY0243).

\section{Supplementary Materials}

Fig 1s: optical images of the contact angle of the CNF/PEG/CA paper TG curves of the CNF/PEG and $\mathrm{CNF} / \mathrm{PEG} / \mathrm{CA}$ paper. (Supplementary Materials)

\section{References}

[1] Z. C. Jiang, Y. Y. Xiao, Y. Kang, B. J. Li, and S. Zhang, "SemiIPNs with moisture-triggered shape memory and self-healing properties," Macromolecular Rapid Communications, vol. 38, no. 14, article 1700149, 2017.

[2] N. Li, G. Chen, W. Chen et al., "Multivalent cations-triggered rapid shape memory sodium carboxymethyl cellulose/polyacrylamide hydrogels with tunable mechanical strength," Carbohydrate Polymers, vol. 178, pp. 159-165, 2017.

[3] Y. Fang, Y. Ni, B. Choi et al., "Chromogenic photonic crystals enabled by novel vapor-responsive shape-memory polymers," Advanced Materials, vol. 27, no. 24, pp. 3696-3704, 2015.

[4] Y. Fang, Y. Ni, S. Y. Leo et al., "Direct writing of threedimensional macroporous photonic crystals on pressureresponsive shape memory polymers," ACS Applied Materials \& Interfaces, vol. 7, no. 42, pp. 23650-23659, 2015.

[5] C. H. Lu, W. Guo, Y. Hu, X. J. Qi, and I. Willner, "Multitriggered shape-memory acrylamide-DNA hydrogels," Journal of the American Chemical Society, vol. 137, no. 50, pp. 15723-15731, 2015.

[6] A. Kausar, "Nanodiamond tethered epoxy/polyurethane interpenetrating network nanocomposite: physical properties and thermoresponsive shape-memory behavior," International Journal of Polymer Analysis and Characterization, vol. 21, no. 4 , pp. 348-358, 2016.

[7] X. Li, T. Liu, Y. Wang et al., "Shape memory behavior and mechanism of poly(methyl methacrylate) polymer networks in the presence of star poly(ethylene glycol)," RSC Advances, vol. 4, no. 37, pp. 19273-19282, 2014. 
[8] D. Ratna and J. Karger-Kocsis, "Shape memory polymer system of semi-interpenetrating network structure composed of crosslinked poly (methyl methacrylate) and poly (ethylene oxide)," Polymer, vol. 52, no. 4, pp. 1063-1070, 2011.

[9] L. N. Woodard, V. M. Page, K. T. Kmetz, and M. A. Grunlan, "PCL-PLLA semi-IPN shape memory polymers (SMPs): degradation and mechanical properties," Macromolecular Rapid Communications, vol. 37, no. 23, pp. 1972-1977, 2016.

[10] L. Zhang, H. Jiao, H. Jiu, J. Chang, S. Zhang, and Y. Zhao, "Thermal mechanical and electrical properties of polyurethane/(3-aminopropyl) triethoxysilane functionalized graphene/epoxy resin interpenetrating shape memory polymer composites," Composites: Part A, vol. 90, no. 1, pp. 286-295, 2016.

[11] R. Zarnetta, R. Takahashi, M. L. Young et al., "Identification of quaternary shape memory alloys with near-zero thermal hysteresis and unprecedented functional stability," Advanced Functional Materials, vol. 20, no. 12, pp. 1917-1923, 2010.

[12] Y. Li, H. Chen, D. Liu, W. Wang, Y. Liu, and S. Zhou, "pHresponsive shape memory poly(ethylene glycol)-poly( $\varepsilon$-caprolactone)-based polyurethane/cellulose nanocrystals nanocomposite," ACS Applied Materials \& Interfaces, vol. 7, no. 23, pp. 12988-12999, 2015.

[13] I. Dueramae, M. Nishida, T. Nakaji-Hirabayashi, K. Matsumura, and H. Kitano, "Biodegradable shape memory polymers functionalized with anti-biofouling interpenetrating polymer networks," Journal of Materials Chemistry B, vol. 4, no. 32, pp. 5394-5404, 2016.

[14] Y. Feng, H. Zhao, L. Jiao, J. Lu, H. Wang, and J. Guo, "Synthesis and characterization of biodegradable, amorphous, soft IPNs with shape-memory effect," Polymers for Advanced Technologies, vol. 23, no. 3, pp. 382-388, 2012.

[15] R. E. Abou-Zeid, E. A. Hassan, F. Bettaieb, R. Khiari, and M. L. Hassan, "Use of cellulose and oxidized cellulose nanocrystals from olive stones in chitosan bionanocomposites," Journal of Nanomaterials, vol. 2015, Article ID 687490, 11 pages, 2015.

[16] L. Song, Y. Li, Z. Xiong et al., "Water-induced shape memory effect of nanocellulose papers from sisal cellulose nanofibers with graphene oxide," Carbohydrate Polymers, vol. 179, no. 1, pp. 110-117, 2018.

[17] J. S. Yeo and S. H. Hwang, "The effect of dense polymer brush on the microfibrillated cellulose for the mechanical properties of poly( $\varepsilon$-caprolactone) biocomposites," International Journal of Adhesion and Adhesives, vol. 78, pp. 89-94, 2017.

[18] E. A. Hassan, S. M. Fadel, and M. L. Hassan, "Influence of TEMPO-oxidized NFC on the mechanical, barrier properties and nisin release of hydroxypropyl methylcellulose bioactive films," International Journal of Biological Macromolecules, vol. 113, no. 1, pp. 616-622, 2018.

[19] S. Fujisawa, T. Saito, S. Kimura, T. Iwata, and A. Isogai, "Surface engineering of ultrafine cellulose nanofibrils toward polymer nanocomposite materials," Biomacromolecules, vol. 14, no. 5, pp. 1541-1546, 2013.

[20] P. Yu, H. He, and A. Dufresne, "A novel interpenetrating polymer network of natural rubber/regenerated cellulose made by simple co-precipitation," Materials Letters, vol. 205, no. 1, pp. 202-205, 2017.

[21] W. S. Abo-Elseoud, M. L. Hassan, M. W. Sabaa, M. Basha, E. A. Hassan, and S. M. Fadel, "Chitosan nanoparticles /cellulose nanocrystals nanocomposites as a carrier system for the controlled release of repaglinide," International Journal of Biological Macromolecules, vol. 111, no. 20, pp. 604-613, 2018.
[22] M. Kaushik, K. Basu, C. Benoit, C. M. Cirtiu, H. Vali, and A. Moores, "Cellulose nanocrystals as chiral inducers: enantioselective catalysis and transmission electron microscopy 3D characterization," Journal of the American Chemical Society, vol. 137, no. 19, pp. 6124-6127, 2015.

[23] M. Morits, J. R. McKee, J. Majoinen et al., "Polymer brushes on cellulose nanofibers: modification, SI-ATRP, and unexpected degradation processes," ACS Sustainable Chemistry \& Engineering, vol. 5, no. 9, pp. 7642-7650, 2017.

[24] M. L. Hassan, J. Bras, E. A. Hassan, S. M. Fadel, and A. Dufresne, "Polycaprolactone/modified bagasse whisker nanocomposites with improved moisture-barrier and biodegradability properties," Journal of Applied Polymer Science, vol. 125, Supplement 2, pp. E10-E19, 2012.

[25] L. Dai and C. L. Si, "Cellulose-graft-poly(methyl methacrylate) nanoparticles with high biocompatibility for hydrophobic anti-cancer drug delivery," Materials Letters, vol. 207, pp. 213-216, 2017.

[26] F. Zhuo, X. Liu, Q. Gao, Y. Wang, K. Hu, and Q. Cai, "Injectable hyaluronan-methylcellulose composite hydrogel crosslinked by polyethylene glycol for central nervous system tissue engineering," Materials Science \& Engineering. C, Materials for Biological Applications, vol. 81, no. 21, pp. 1-7, 2017.

[27] D. Cheng, Y. Wen, L. Wang, X. An, X. Zhu, and Y. Ni, "Adsorption of polyethylene glycol (PEG) onto cellulose nano-crystals to improve its dispersity," Carbohydrate Polymers, vol. 123, no. 1, pp. 157-163, 2015.

[28] P. de Cuadro, T. Belt, K. S. Kontturi et al., "Cross-linking of cellulose and poly(ethylene glycol) with citric acid," Reactive \& Functional Polymers, vol. 90, no. 3, pp. 21-24, 2015.

[29] L. Dong, C. Hu, L. Song, X. Huang, N. Chen, and L. Qu, "A large-area, flexible, and flame-retardant graphene paper," Advanced Functional Materials, vol. 26, no. 9, pp. 14701476, 2016.

[30] J. J. M. Halls, C. A. Walsh, N. C. Greenham et al., "Efficient photodiodes from interpenetrating polymer networks," Nature, vol. 376, no. 6540, pp. 498-500, 1995.

[31] Y. Liu, Y. Li, G. Yang, X. Zheng, and S. Zhou, "Multi-stimulusresponsive shape-memory polymer nanocomposite network cross-linked by cellulose nanocrystals," ACS Applied Materials \& Interfaces, vol. 7, no. 7, pp. 4118-4126, 2015.

[32] H. Du and J. Zhang, "Solvent induced shape recovery of shape memory polymer based on chemically cross-linked poly(vinyl alcohol)," Soft Matter, vol. 6, no. 14, pp. 3370-3376, 2010.

[33] Y. Liu, Y. Li, H. Chen, G. Yang, X. Zheng, and S. Zhou, "Water-induced shape-memory poly(d, 1-lactide)/microcrystalline cellulose composites," Carbohydrate Polymers, vol. 104, no. 1, pp. 101-108, 2014.

[34] W. Wang, H. Lu, Y. Liu, and J. Leng, "Sodium dodecyl sulfate/epoxy composite: water-induced shape memory effect and its mechanism," Journal of Materials Chemistry A, vol. 2, no. 15, pp. 5441-5449, 2014. 


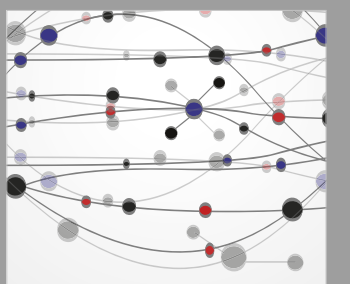

The Scientific World Journal
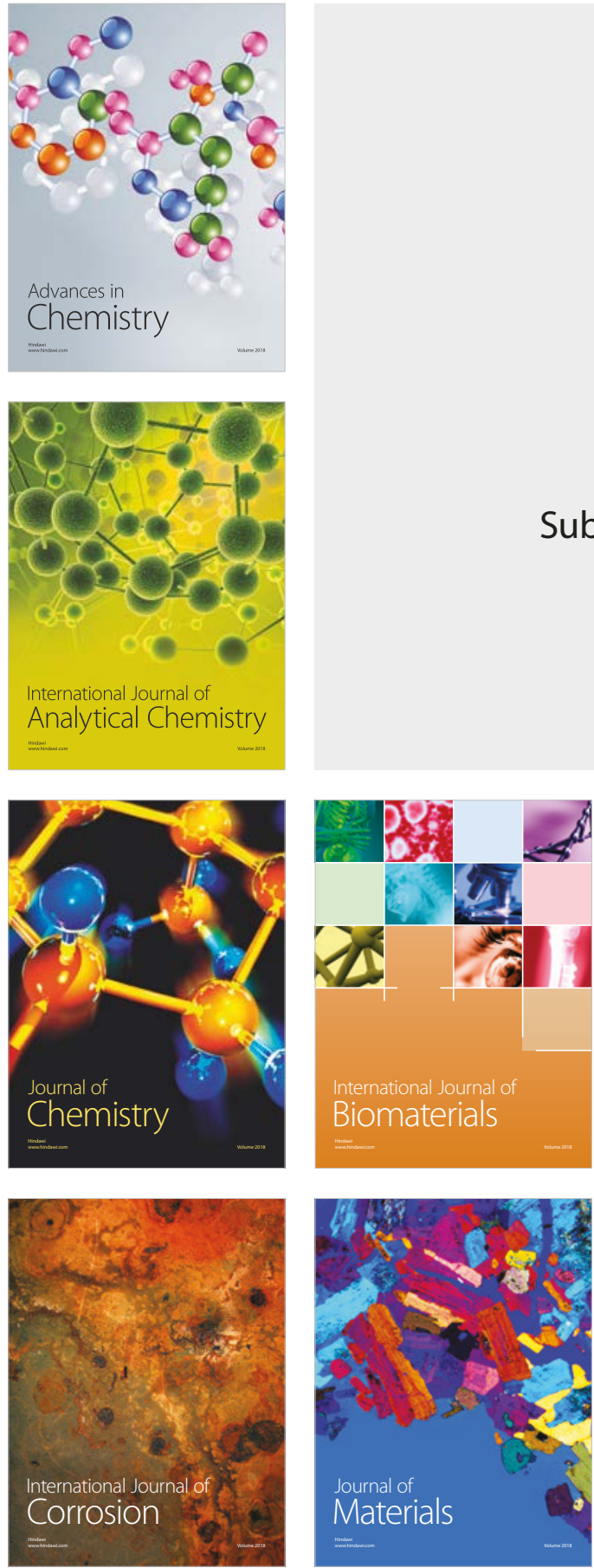

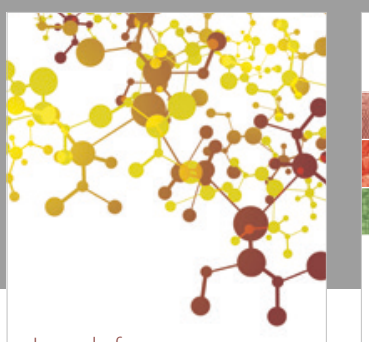

Journal of

Applied Chemistry
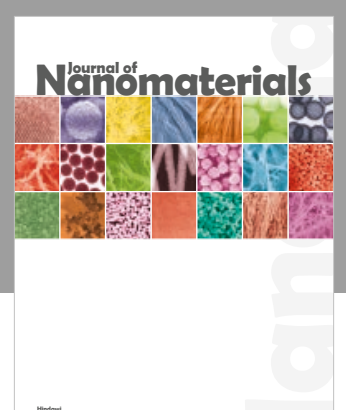

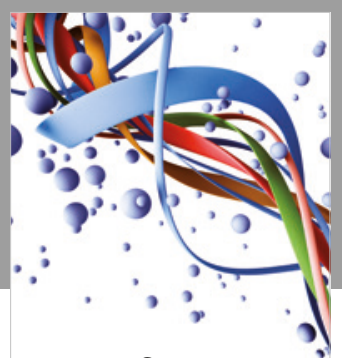

Scientifica

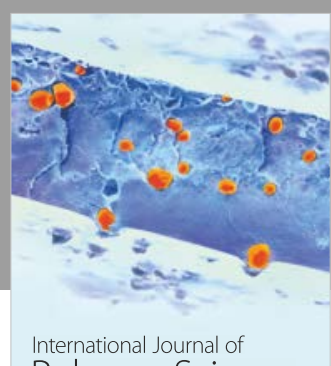

Polymer Science

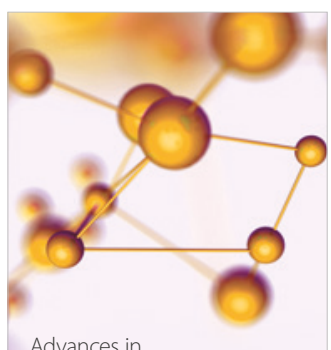

Physical Chemistry
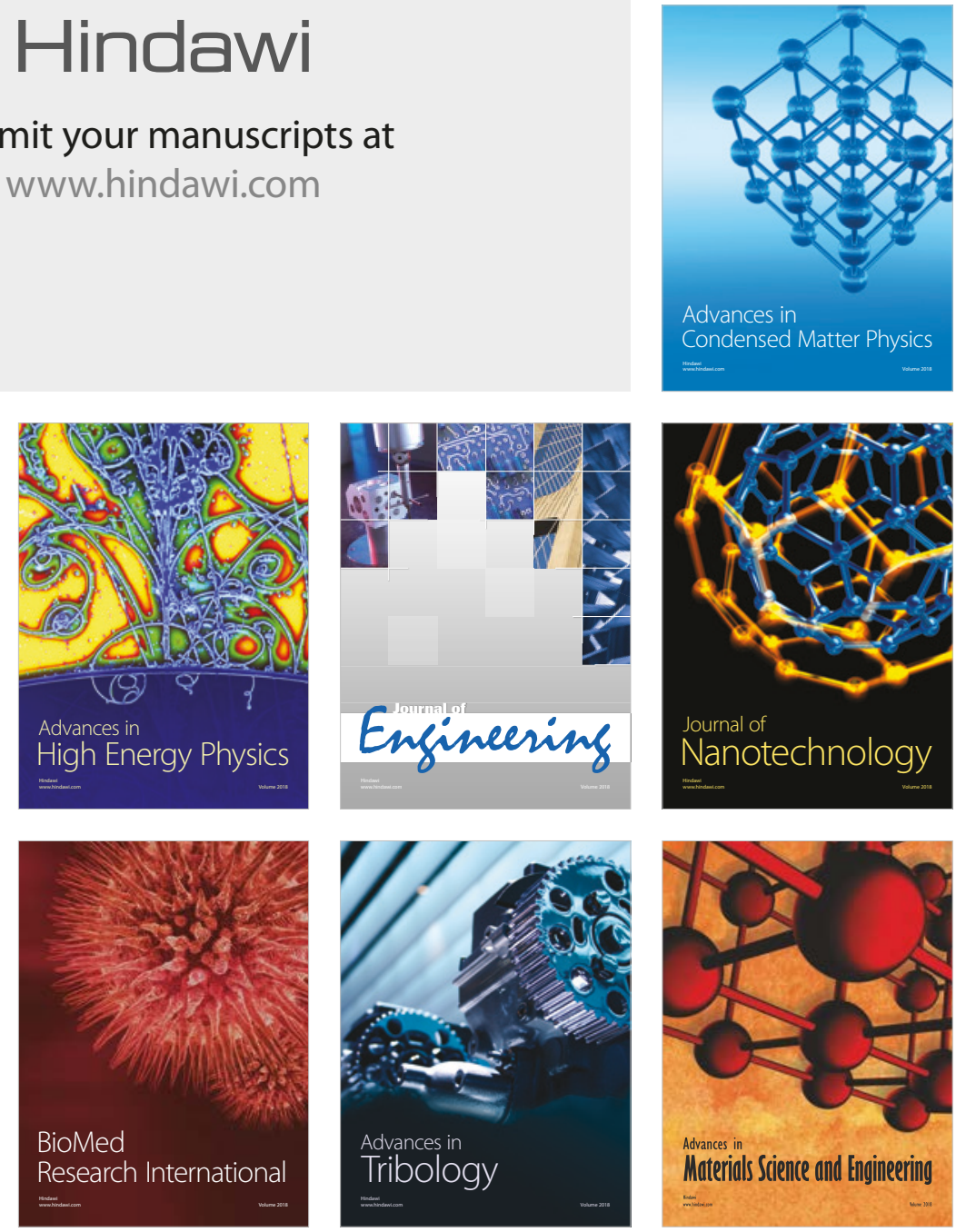